\title{
The Roles of Multilingual Learning Facilitators in Assisting Non-Chinese-Speaking Students in Learning Chinese in Hong Kong: An Exploratory Study
}

\author{
Shek-Kam Tse*, Heiken To, Pik-Fong Tsui, Victor Laing, Kam-Fong Wu, Lu-Sai Lam \\ Faculty of Education, The University of Hong Kong, Hong Kong, China \\ Email: *sktsebrian@gmail.com
}

How to cite this paper: Tse, S.-K., To, H., Tsui, P.-F., Laing, V., Wu, K.-F., \& Lam, L.-S. (2021). The Roles of Multilingual Learning Facilitators in Assisting Non-ChineseSpeaking Students in Learning Chinese in Hong Kong: An Exploratory Study. Open Journal of Social Sciences, 9, 494-509. https://doi.org/10.4236/jss.2021.91035

Received: December 2, 2020

Accepted: January 26, 2021

Published: January 29, 2021

Copyright $\odot 2021$ by author(s) and Scientific Research Publishing Inc. This work is licensed under the Creative Commons Attribution International License (CC BY 4.0).

http://creativecommons.org/licenses/by/4.0/

(c) (i) Open Access

\begin{abstract}
This study aimed to define the roles played by Multilingual Learning Facilitators (MLFs), a novel post created to facilitate non-Chinese-speaking (NCS) pre-schoolers in learning Chinese in Hong Kong, based in community agencies. Qualitative analysis of field observations and interviews with MLFs and social workers found that roles fell under teaching-related and social workrelated categories. For teaching-related roles, MLFs facilitated learning of Chinese in NCS pre-schoolers, assisted social workers in classroom management during activities, engaged NCS parents, and fostered communication between social workers and NCS parents. For social work-related duties, MLFs offered consulting for NCS parents dealing with issues, and also fostered communication between social workers and NCS parents. These roles are made possible due to MLFs' language and cultural expertise of that of NCS families and the local Chinese community. This addresses current limitations faced by teachers and social workers in their roles in teaching NCS students, and expands resources available for NCS families, from schools to the community, in supporting their children in learning Chinese, potentially opening up opportunities for NCS children to integrate into the local community.
\end{abstract}

\section{Keywords}

Multilingual, Multicultural, Non-Chinese Pre-Schoolers, Chinese Learning, Chinese as a Second Language

\section{Introduction}

According to Bronfenbrenner's (1993) Ecological Systems Theory, one should con- 
sider the interconnections of different systems and how they combine to influence children, instead of considering stakeholders and the environment around children as separating from one another. This has given rise to a Home-School-Community (HSC) collaborative approach in child education. The home domain concerns aspects such as parental involvement in children's learning, along with parenting knowledge and skills. The school domain contains aspects such as school policies, educational materials, environment and teaching quality. The community domain considers resources from community agencies available to children, families and schools and how they can be connected to these resources (Smith et al., 1997; Suarez-Orozco, Onaga, \& De Lardemelle, 2010).

Research has found that collaboration between parents, teachers and the community has positive benefits for children academically, emotionally and socially (Pomerantz, Moorman, \& Litwack, 2007; Sheldon, 2007). According to Epstein (1994), this collaboration allows different stakeholders to contribute to shared interests for the bettering of children's academic and personal development. Specifically for the community domain, a study by Sheldon (2003) demonstrated that a higher degree of school involvement with families and community partnership programs was related to better academic achievements in early elementary grades. Wheeler, Guevara and Smith (2018) suggest that community resources have the benefit of providing additional support to students from disadvantaged backgrounds to fulfill their academic potential.

\subsection{Community-Based Language Learning (CBLL)}

Clifford and Reisinger (2019) coined the term community-based language learning, which refers to the use of the community as a partner in the language learning process, which can be understood as one form of involvement of communities in the HSC model of learning. CBLL understands language and language learning as embedded in culture and context. Anderson, Anderson, Hare and McTavish (2015) note that communities provide important social, cultural and linguistic constructs in early childhood (Anderson, Anderson, Hare, \& McTavish, 2015). Grant and Ray (2019) mentions the importance of schools to collaborate with community agencies familiar with specific populations, especially when there exists language and cultural differences between homes and schools, as these agencies are best equipped to provide relevant contexts for children when learning. Benefits of CBLL in second language (L2) learning include increased motivation, positive attitudes toward language learning, willingness to use the L2 language, and increased confidence in using the L2 language (Clifford \& Reisinger, 2019; Pellettieri, 2011; Wehling, 2011; Pak, 2007).

\subsection{Challenges Faced by Non-Chinese-Speaking (NCS) Students in Learning Chinese in Hong Kong}

For clarity, Chinese in this study refers to a combination of spoken Cantonese, a dialect of Chinese, together with written Chinese. Massing, Kirova and Hennig's 
(2013) noted reasons for immigrant families being less involved in their children's learning included linguistic differences, lack of support and lack of time and material resources. Support provided by the local government for NCS students in learning Chinese in Hong Kong is currently limited. Websites operated by the Education Bureau list the educational services available for NCS students (Education Bureau [EDB], 2019a). A Student Support Programme is also available to help NCS students learn Chinese at Chinese Learning Support Centres operated in various districts with after-school activities (Education Bureau [EDB], 2015). However, these efforts mainly assist NCS students in primary and secondary schools. The assistance to NCS students' parents is also far from enough, limited to an e-version of parent information package for NCS parents (Education Bureau [EDB], 2019b).

Policy-wise, NCS students had to attend designated schools up until 2003, when the Equal Opportunity Commission (EOC) recommended earlier integration of NCS students into the local community (EOC, 2011). Since then, however, schools with high proportions of NCS students have remained, with around $40 \%$ of NCS students studying in schools consisting of $70 \%$ or more of NCS students in their population (EOC, 2019). This means that NCS students are still relatively enclosed within their communities, with inadequate exposure and interactions using the Chinese language and with the local culture.

Both the EOC (2019) and Tsung and Gao (2012) note that cultural and language differences pose challenges for NCS students in learning Chinese. Differences in culture mean that NCS students may spend most of their time with members of their own culture, isolating themselves from the local community and limiting opportunities to practice Chinese, as well as exposure to cultural contexts of the language. Written Chinese is also logographic in nature, in contrast to alphabetical systems used in many native languages of NCS students, such as Urdu and Nepali. Parents of NCS students also suffer from the cultural and language barrier due to insufficient knowledge of Chinese and the local culture, meaning that they are not able to provide enough support for their children, and are more likely to be less involved in their children's language learning.

\subsection{Limitations of Kindergarten Teachers' and Social Workers' Capacity in Supporting NCS Students in Hong Kong}

Kindergarten teachers worldwide face considerable workloads, with most stretched to the limit of their capacities. In a study conducted by Richards (2012) which included kindergarten teachers, the numerous duties and responsibilities, along with inadequate support in teaching students with special needs, formed the top two reasons for workplace stress. In another study, Dibbon (2004) identified other sources of stress in teachers, including lack of time for preparation and work, large classes with diverse needs, and lack of resources in supporting implementation of programs. A similar situation is observed in Hong Kong. According to a survey conducted by the Hong Kong Federation of Education Workers (2019), among 1200 kindergarten teachers, $45 \%$ work for 61 hours or more 
per week, with more than $90 \%$ of teachers reporting work-related stress, to the point that $42 \%$ are considering quitting the education sector within the next two years. The majority of teachers claimed a lack of resting time, as well as heavy administrative workloads.

With kindergarten teachers already operating at their limits, it would be demanding and infeasible to require them to take up further responsibilities involving both families and the community, and receive additional training for cultural competence, in accordance to the HSC model. Specifically, for working with NCS students and their families, even for current teachers of NCS students, it is not a given that they understand the cultures of NCS students, let alone know their languages. NCS students' parents may speak limited English, and as aforementioned, this language barrier hampers teachers from forming good partnerships with parents. One suggestion would be to introduce social workers from agencies that serve NCS populations. However, social workers are not mandated in kindergartens currently in Hong Kong, and most kindergartens lack support from social worker support, due to lack of funding (Joint Committee concerning Kindergarten-Based Social Workers, 2018). Also, most social workers in these agencies are local Chinese, and like the teachers, may not be familiar with the languages of NCS students and their parents.

\section{The Current Study}

Past research has provided evidence for the use of the HSC model in improving language learning of students, and specifically within the community domain, the CBLL approach emphasizes the use of communities in the L2 language learning process to provide social, cultural and linguistic contexts. However, given the current capacity of teachers and social workers in Hong Kong kindergartens, together with challenges faced by NCS students in learning Chinese, there exists a need for a facilitator between families, schools and community agencies if the HSC model is to be effectively implemented. The role calls for knowledge and experience in the local language and culture, as well as that of the NCS population.

In view of this, the post of multilingual learning facilitator (MLF) was created as part of the C-for-Chinese@JC project, based in community agencies already serving the NCS population. In the original design, MLFs are expected to execute teaching-related and social work-related duties. For teaching duties, MLFs work alongside social workers in community activities designed to teach Chinese, embedded in the local context, to NCS students from affiliated kindergartens. For social work-related duties, MLFs provide interpretations culturally and linguistically when communicating with parents, social workers and teachers, enabling the parties to form collaborations with better understanding of one another.

The current study aims to investigate the duties of MLF in real practice in order to better define the roles of MLF in facilitating learning in NCS students. With better definition of roles, it may provide insight into how this post may be practically integrated into existing scenarios at homes, schools and communities 
in the future. The study adopts an exploratory case study approach, and consists of two parts. The first part concerns the teaching aspect of MLFs, whereas the second focuses on the social work aspect.

\section{Methodology}

\subsection{Study 1}

\subsubsection{Participant and Procedures}

Study 1 was a case study involving an observation with video of a Chinese learning class conducted in a community agency, and a semi-structured interview. The Chinese learning class was led by a Chinese, female social worker and assisted by a female MLF, who was a Pakistani, with 7 NCS attendees and their parents. The class was held in November 2017 and the content of that day was teaching of Chinese words of body parts to NCS students. All the participants were notified about the video shooting. After the class, a semi-structured interview was conducted by a research staff from the "C-for-Chinese@JC" project with the social worker and the MLF. The interview lasted for 20 minutes. Consent was obtained prior to the data collection.

\subsubsection{Data Analysis}

For both the observation and the interview, thematic analysis was used. For the field observation, the conversations, as well as actions of the social worker, the MLF, students, and parents, were transcribed. The interview was transcribed verbatim. Afterwards, simple coding was done by a trained rater, then cross-checked by another rater for interrater reliability. Themes agreed to both raters were then further coded and extracted.

\subsubsection{Results-Field Observation}

\section{Facilitate learning of Chinese in NCS students}

Analysis of the community activity revealed three main roles played by MLFs. One role was to facilitate overall learning of NCS students. Within this role, three teaching skills were observed to be used by the MLF: use of verbal and non-verbal instruction, questioning, and positive reinforcement. The following example illustrates the use of the three skills:

\begin{tabular}{ccc}
\hline $\begin{array}{c}\text { Social worker: } \\
\text { Social worker \& MLF: }\end{array}$ & This is the nose, follow me by saying "nose". & \\
Students: & Nose. & Verbal instruction \\
Nocial worker: & $\begin{array}{c}\text { What about this? (Shows card with a } \\
\text { picture of a pair of eyes) }\end{array}$ & \\
Students: & Eyes. & Questioning \\
MLF: & Where are your eyes? & \\
Students: & Eyes. (Points to own eyes) & Positive reinforcement \\
MLF: & Good girl. &
\end{tabular}


The following is another example illustrating the use of non-verbal instructions:

Social worker:
Now let's circle the word "ear"
Circle the ear one... can you see it?
(Points to the worksheet)
Social worker:
Where is the word "ear?"
(Some students circle the word)

The verbal and non-verbal instructions used by the MLF were mainly prompting in nature, repeating the instructions of the social worker, but also giving cues to the students, such as pointing at the worksheet to allow them to better understand what they needed to do. Questioning served the purpose of elaborating on the social worker's teaching, such as asking students where their eyes were, after the social worker mentioned the word "eyes". It was also used in clarification, to check if the students understood the task at hand, such as asking them if they saw the Chinese word "ear". Positive reinforcement in the form of praises was given when the students accomplished the task, rewarding them and motivating them for further learning.

In addition to teaching skills, the MLF was also observed to use her expertise in the native language of NCS students to provide interpretations of the social worker's instructions. This again served the purpose of allowing NCS students to better understand what they need to do during the activities. Here is an example in an activity where NCS students were instructed to complete a jigsaw puzzle:

Social worker: $\quad \begin{gathered}\text { Good. Now let's find the other half of the puzzle. Find } \\ \text { your classmate that holds the other half of your puzzle. }\end{gathered}$
MLF:
(Translates in Urdu)
Social worker: $\quad \begin{gathered}\text { (Points to the puzzle piece) Yours is a pizza. Where is } \\ \text { the other piece? Let's see if this is the one. } \\ \text { MLF: } \\ \text { (Translates in Urdu) }\end{gathered}$ Interpretation
(Students successfully finished the puzzle)

Assist the social worker in classroom management

The MLF was observed to assist the social worker in carrying out activities, such as distributing worksheets and pens. The following is an example:

$\begin{gathered}\text { Let's have a look. Last time, we did not finish the worksheet. } \\ \text { Let's complete it today. }\end{gathered}$
(MLF distributes worksheets and pens)
Assisting social
worker




\section{Engage NCS parents}

The MLF was also seen to engage parents during the activities with the NCS students. Engaging parents allows them to keep track of their children's learning content and progress, so that they may be able to assist their children in learning Chinese outside of classes. In this activity, the MLF engages both the parent and student when learning the Chinese word for "hand":

Social worker: Yes, this is the word for "hand". We need to circle this one.
MLF: $\quad \begin{aligned} & \text { Okay, mama will help. This one is the word for "hand". } \\ & \text { (Turns to students). Is this the word for hand? }\end{aligned}$

\subsubsection{Results-Semi-Structured Interview}

The semi-structured interview revealed four main roles of MLFs in teaching-related duties:

Facilitate learning of Chinese in NCS students

The social worker noted that having an MLF that was familiar with the language of NCS students was important in her teaching, as students may not understand her instructions completely, and that the MLF was helpful in explaining the instructions to them in their own native language so that students would be able to follow the lesson progress:

"Some children only understand 50\%, so it would be helpful to have a Pakistani colleague to help, because some children really don't understand instructions, if someone can remind them, it would be easier for the children to follow the lesson progress."

The social worker also mentioned that the expertise of the MLF in NCS cultures helped her in designing culturally-appropriate and relevant activities for NCS students, allowing them to learn Chinese in a way in which they could relate to their own background and experiences:

"She (the $M L F)$ can give some suggestions of activities... I $m$ not an expert of the Pakistani culture, so she can tell me for example, what is the Pakistani parenting style, then I can have some more information."

Assist the social worker in classroom management

The MLF was seen to assist students who needed more help in learning Chinese, bridging the gap in learning differences of students. As the MLF expressed:

"Instead of helping him (a more able student) I will help others that need more help."

The MLF also noted briefly that classroom management was part of her duties during activities, including crisis management, such as when students cry, or when behavioural issues arise:

"They (children) get noisy and distracting others, so I say "stay with me, stay with me." 
Foster communication between the social worker and NCS parents

The MLF mentioned that since NCS students and their parents may not understand certain words and sentences in Chinese, she plays a role in interpreting what the social worker says to the parents in their native language, so they can better understand what the intentions of the social worker:

"I make the communication easier, there are some words that they cannot understand in Chinese, so I interpret for her when she (the social worker) starts talking to her (parent)."

Interpretation has to be done in a culturally-relevant manner for both parties to understand each other well. The MLF, in particular, noted that her familiarity with facial expressions and body language specific to NCS cultures was helpful in allowing her to understand hidden thoughts and concerns of NCS parents, and convey them to the social worker accordingly:

"For me I operate between social workers and parents, so far P ve tried my best and I am kind of satisfied with that... I make sure I know what they are thinking... because from their facial expression and body language I can understand what is going on in their brains... I don't know how to explain but I can feel it."

The social worker added that it was a bonus to have a colleague alongside that was from the background of NCS families, as it gave a sense of familiarity to the parents, potentially allowing them to open up more easily for better communication, and in turn, for social workers to build partnerships with them:

"I think there is a difference if I brought along a Chinese colleague versus a Pakistani colleague... when parents see her (the MLF) then they will feel better... at least they can communicate with her."

Engage NCS parents

Given her familiarity with both Chinese and Urdu, coupled with the experience of learning Chinese as an NCS in Hong Kong when she was young, the MLF noted that she understood the difficulties involved. She saw parents as a resource for their children, and provided Urdu pronunciations of Chinese words for them to practice with their children at home:

"Parents tell me 'We can't remember things when we go home, so what should we do? Because I am also an EM, when I studied I also understood the difficulty, so I asked them to make a notebook, and then write the pronunciation in Urdu, and the meaning, and try to practice."

"Some mothers cannot read English, so they ask me to write Urdu for them."

\subsection{Study 2}

\subsubsection{Participant and Procedures}

Study 2 was a semi-structured interview with four MLFs, all were Pakistani and 
female. The interview was taken place in December 2019 after the MLFs having served the community agency from half a year to a year, aiming to explore possible roles played in social work-related duties, from the perspective of MLFs. Consent was obtained prior to the data collection.

\subsubsection{Data Analysis}

A thematic coding approach was used. The interview was transcribed verbatim. Afterwards, simple coding was done by two trained experts, then cross-checked for interrater reliability. Common themes were extracted and presented below.

\subsubsection{Results}

From the interview, the following roles of MLFs were identified in the area of social work-related duties:

Offer consulting for issues faced by NCS parents

Having been brought up in the NCS culture, but living in Hong Kong, all MLFs mentioned that their experience in tasks such as filling in forms, dealing with schooling and housing issues meant that they could help NCS parents navigate similar issues in daily life. This was especially true given that most parents may not know Chinese or even English, and that mothers from NCS cultures stay at home most of the time. In other words, MLFs were able to help these parents solve daily life issues brought by the language and cultural barrier:

"Many women don't leave home often... they can ask us for help as they do not know, for example, how to fill in forms."

"We all help parents fill in forms, like in housing, as their family members may not know Chinese or English."

"We grew up in Hong Kong, so we have the experience when we were young of filling in forms or applying for schools for ourselves or our siblings, for example."

"If they do not know things such as how to choose schools, we can help them and teach them what to do. We can also help, for example, if they need to apply for things."

Another issue arising with the language and cultural barriers is that parents of NCS families may not be updated with the local news. Thus, MLFs also informed the NCS parents on news and things that they need to be aware of:

"We also update them on the local news, on what they need to be aware of, and on resources from the community... because most mothers go out rarely and don't know Chinese."

For issues out of MLFs' expertise, they would refer parents to appropriate support services:

"For other problems, we (community agency) have the expertise from members, such as social workers, in helping them out."

"We take an active role in helping them. If there are problems we cannot 
resolve then we can always find help."

Foster communication between social workers and NCS parents

By playing the role of an interpreter, MLFs could address the language barrier between NCS families and social workers, meaning that parents were able to convey their needs, and in turn, social workers were able to more effectively help them address these needs:

"We build relationships with parents and understand their needs, because we know different languages. With this ability, we can help parents, they can find us for help anytime, then we can find out their needs."

"If they need help from teachers or social workers, we can help them ask... because we know their language."

In addition, MLFs were able to bridge cultural differences in communication between social workers and NCS parents:

"We are like a middle person, we build relationships with parents... when they see that we are Pakistani, they can tell us about what's happening in their homes, or if they have something they don't know about the community, they will share with us."

MLFs revealed that enhancing the relationships between NCS parents and social workers made NCS parents view community agencies as a trustworthy source of help in their daily issues, and they were more likely to reach out for help, as evident by the following quotes:

"We have seen parents living here for 15 - 20 years, but have never received such services. Parents sometimes don't trust older siblings in the family, so may ask us first."

"Somebody what sapped us one time, wanting to speak to us, but we didn't know that person... turns out it was a friend of a mother who used our services before, and the mother had sent the contact number to her friend... her friend's son was just going into $K 1$ and asked us if there are any Chinese classes offered at our centre."

\section{Discussion}

The present study explored a culturally responsive role, MLF, as proposed in a Chinese enhancement program for NCS students. Using field observation and semi-structured interviews, MLFs' role in assisting the learning of Chinese of NCS students by playing their teaching-related and social work-related roles are clearly defined as shown in Figure 1.

There are five main roles within teaching-related duties of MLFs; the first was facilitating learning of Chinese in NCS students. This involves the use of teaching skills during activities, such as verbal and nonverbal instructions, questioning, and positive reinforcement. MLFs also act the role as interpreter during activities as they know the native language of the NCS students. This is particularly 


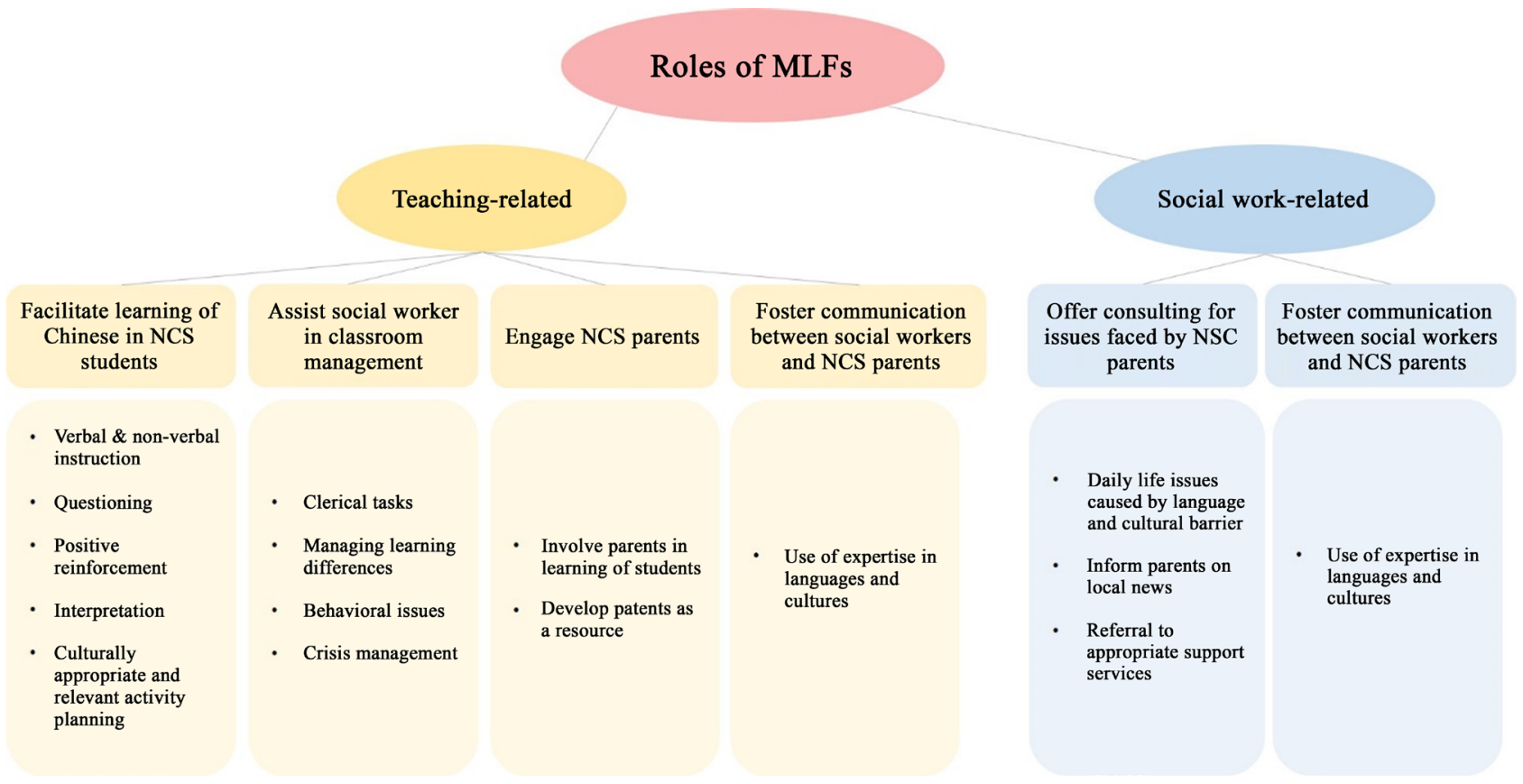

Figure 1. Teaching and social work-related roles of MLFs.

useful for students who are new to learning Chinese, and they require more assistance in explanation using their own native language. The use of teaching skills and interpretation allows NCS students to get a better understanding of the content being taught and know what they need to do during activities, with the implications being that they are likely to be more involved in the activities, and likely to retain new knowledge learnt about the Chinese language.

MLFs also facilitate learning of Chinese in NCS students by contributing to the planning of culturally appropriate and relevant activities. MLFs inform social workers on, for example, customs, traditions, parenting styles of NCS families, and potential pitfalls that people from these cultural backgrounds may find offensive. In turn, social workers are more likely to deliver culturally appropriate activities and make use of NCS students' experiences of their native languages and culture to that of the local community.

The second role MLFs perform is assisting social workers in classroom management. This ranges from simple clerical tasks, such as distributing worksheets, to aspects required specific skills, such as managing learning differences, behavioural management, and crisis management. These all serve to free up the capacity of social workers, so they can focus on leading the activity with minimal interruption. Students who need more assistance in learning also benefit by getting additional attention from the MLF.

Having the background in the languages and cultures of NCS families and local Chinese, MLFs are well-positioned to play the third role of fostering communication between social workers and NCS parents. Acting as a mediator between the two, MLFs provide interpretations, removing not only the language barrier, but also cultural barriers such as differences in parenting styles and cul- 
ture-specific body language. The familiarity of a person from the same background also allows NCS parents to open up more easily and reach out for help when needed. A strong relationship is more likely to result as both parties can have a good understanding of the needs, concerns and intentions of each other.

Naturally, MLFs also play the role of engaging with NCS parents. This is mainly done to involve parents in the learning process of their children, and to equip parents with relevant knowledge and skills to help their children learn outside of the classroom. In particular, the experience of growing up from an NCS family in Hong Kong allows MLFs to anticipate similar challenges of learning Chinese faced by NCS students, and offer suggestions and support to their parents, such as providing pronunciations of Chinese words in their native language. In turn, parents are able to practise with their children at home and further support their learning.

For social work-related duties of MLFs, the research results revealed two main roles. The first is a consulting role to help NCS parents address issues faced in daily life, arising from the language and cultural barrier. MLFs, drawing upon their experience of growing up in Hong Kong, are able to offer advice for parents in filling in forms, applying for housing and schools, etc. They also update parents on local news and alert them on issues that may affect them. For issues outside of their abilities, MLFs can still make use of their language expertise in referring parents to other support services, such as those given by social workers and psychologists. Secondly, MLFs also play a role in fostering communication between members from support services, such as social workers, and NCS parents. When NCS parents tell MLFs about the issues they are encountering, MLFs are able to convey not only the issues, but also the cultural context of the issues to social workers, so that they gain a more rounded understanding on how to help the parents. Considering the two roles played by MLFs, the implications are that parents will be more likely to reach out to resources in the community for help, in addition to those offered at schools, meaning that their children indirectly benefit from the extra support.

Looking at the roles for both teaching and social work-related duties of MLFs, it can be seen that apart from assisting social workers in classroom management, all other roles involve the contribution of MLFs' language and cultural expertise. This is similar to the results from a study by Massing et al. (2013), where language facilitators in kindergarten classrooms were found to bring first-hand knowledge of 'families' cultural traditions and the realities of their day-to-day lives" (p. 8), providing accurate interpretations of students' actions given their cultural background, where local teachers may otherwise miss or misinterpret. The use of students' native languages is also found to be helpful in helping their language learning, and allowing parents to feel more welcomed in the classroom. Considering that existing research on teaching and learning of Chinese as a second language has mainly focused on the practices of teaching and the processes of acquisition and learning, this study also highlights the importance of considering 
socio-cultural contexts in supporting the learning of Chinese as a second language, especially when learners are living in an environment with Chinese as the dominant language.

In the context of the HSC model of learning (Grant \& Ray, 2019), the use of MLFs directly increases the resources available from the community domain, providing expanded capacity in teaching and social work-related services that previously could not be achieved due to the limitations in kindergarten policies, as well as breaking the language and cultural barrier that existed between local Chinese and NCS cultures. Given the expertise of MLFs in the languages and cultures of both sides, they also greatly facilitate CBLL by providing context to both social workers and NCS students in the teaching and learning of Chinese as a second language. This allows NCS students to learn how Chinese is embedded and practically used in daily life, as opposed to learning the language on its own. Together with the fact that the learning activities are placed in real-life settings, it provides more exposure for NCS students to the local Chinese culture, potentially facilitating a smoother integration into the local community.

The benefits of MLFs also have a ripple effect to the home and school domains. As MLFs engage NCS parents and foster communication between social workers and NCS parents, the home-community collaboration is strengthened, with parents both receiving more resources in supporting their children in learning, such as knowing how to apply for suitable schools, and at the same time empowered themselves to support their children, such as practising Chinese with their children at home. In other words, by breaking the language and cultural barriers, MLFs help NCS parents to be more involved in the learning progress of their children, potentially allowing for better learning outcomes.

For the school domain, as aforementioned, teachers are often working at their capacity, meaning that they may not have the time and resources to connect with members from the home and community domains. They also face the language and cultural barrier when communicating with NCS families, which requires additional training to overcome. With MLFs, they are able to fill this gap without overburdening teachers.

While the MLF post was created and trialed for the 20 local kindergartens participating in the current study, it remains to be seen how such a post could be practically established and implemented in the wider kindergarten sector. The most obvious approach would be to create an officially recognized post in kindergarten policies, along with funding from the government, which would encourage kindergartens with NCS students to adopt services from MLFs. Such a post would naturally require MLFs to meet certain standards. To achieve this, training could be provided from tertiary institutions with accredited programs, similar to existing teacher training programs.

As the MLF post is cross-disciplinary in nature, one important consideration is the balance of teaching or social-work-related duties of MLFs. This depends on the needs of NCS families, their schools, and related community agencies. It 
also guides whether funding is provided by the local education bureau or the social welfare department. While the MLFs in this study are based in community agencies, the option of school-based MLFs, employed by community agencies, is also worth further exploring. With this mode of operation, MLFs may be able to work with teachers and school-based social workers more closely, follow the learning progress of NCS students at schools, while also retaining a link to the agencies that are familiar with providing supporting services to NCS families. With a greater emphasis on teaching-related duties, additional training in teaching may be considered for better collaboration between MLFs and teachers.

Alternatively, the MLF post could be viewed as a career pathway with two routes, one leading to a position as a teacher, and the other as a social worker. Their experiences as an MLF will greatly inform them in the areas of kindergarten education and social welfare practices, and together with their expertise in the languages and cultures of NCS families, will make them specialized teachers and social workers that bring greater impact to NCS families.

This study has identified possible roles of the novel MLF post in linking home, school and community domains in NCS students, addressing existing challenges faced by NCS students in learning Chinese in Hong Kong, as well as the limitations of teachers and social workers. The study is mainly exploratory as the post is at its beginning, so future studies could be replicated in a larger scale, and further refines the roles played by MLFs and how they may integrate into homes, schools and communities to better the learning of Chinese in NCS students in Hong Kong. Outside of Hong Kong, such a post may also be adapted for use, where learning takes place involving educators, community agencies, students and their families from more than one language and cultural background.

\section{Funding/Financial Support}

This work and the "C-for-Chinese@JC" project were supported by The Hong Kong Jockey Club Charities Trust.

\section{Conflicts of Interest}

The authors declare no conflicts of interest regarding the publication of this paper.

\section{References}

Anderson, A., Anderson, J., Hare, J., \& McTavish, M. (2015). Research with Young Children and their Families in Indigenous, Immigrant, and Refugee Communities. In O. Saracho (Ed.), Handbook of Research Methods in Early Childhood Education (Vol. 2, pp. 115-145). Charlotte, NC: Information Age Publishing.

Bronfenbrenner, U. (1993). The Ecology of Cognitive Development: Research Models and Fugitive Findings. In R. H. Wozniak, \& K. W. Fischer (Eds.), Development in Context: Activity and Thinking in Specific Environments (pp. 3-44). Mahwah, NJ: Lawrence Erlbaum.

Clifford, J., \& Reisinger, D. (2019). Community-Based Language Learning: A Framework 
for Educators. Washington DC: Georgetown University Press. https://doi.org/10.2307/j.ctv7cjw41

Dibbon, D. (2004). It's about Time! A Report on the Impact of Workload on Teachers and Students.

http://files.nlta.nl.ca/wp-content/uploads/public/documents/wrkldstudy rprt/wrkldrpr t04.pdf

Education Bureau, HKSAR (2015). Chinese Language Learning Support Centres. https://www.edb.gov.hk/en/student-parents/ncs-students/support-to-parents-and-stud ents/Chinese\%20Language\%20Learning\%20Support\%20Centres.html

Education Bureau, HKSAR (2019a). Education Services for Non-Chinese Speaking (NCS) Students.

https://www.edb.gov.hk/en/student-parents/ncs-students/about-ncs-students/index.ht $\underline{\mathrm{ml}}$

Education Bureau, HKSAR (2019b). Non-Chinese Speaking Parent Information Package: Your Guide to Education in Hong Kong-e-Version.

https://www.edb.gov.hk/en/student-parents/ncs-students/support-to-student-and-pare nts/package/ncs-parent-information-package-chi eng.html

Epstein, J. L. (1994, E-book in 2019). Theory to Practice: School and Family Partnerships Lead to School Improvement and Student Success. In B. Werber, \& C. Fagnano (Eds.), School, Family, and Community Interaction: A View from the Firing Lines (pp. 39-52). London: Routledge. https://doi.org/10.4324/9780429305375-4

Equal Opportunities Commission (EOC) (2011). Education for All-Report of the Working Group on Education for Ethnic Minorities. http://www.eoc.org.hk/EOC/Upload/UserFiles/File/EducationReportE.pdf

Equal Opportunities Commission (EOC) (2019). Closing the Gap: Report of the Working Group on Education for Ethnic Minorities. https://www.eoc.org.hk/s/ClosingtheGap/Closing the Gap Report.pdf

Grant, K. B., \& Ray, J. (2019). Home, School, and Community Collaboration: Culturally Responsive Family Engagement (4th ed.). Thousand Oaks, CA: SAGE Publications, Inc.

Hong Kong Federation of Education Workers (2019). Kindergarten Teachers Face Long Hours and Great Pressure: More than 40\% of Teachers Considering Leaving the Industry. https://hkfew.org.hk/UPFILE/ArticleFile/201952816193223.pdf

Joint Committee concerning Kindergarten-Based Social Workers (2018). Kindergarten Social Worker Pioneering Project Letter of Recommendation.

https://www.legco.gov.hk/yr17-18/chinese/panels/ws/papers/ws20180622cb2-1669-1-c. pdf

Massing, C., Kirova, A., \& Hennig, K. (2013). The Role of First Language Facilitators in Redefining Parent Involvement: Newcomer Families' Funds of Knowledge in an Intercultural Preschool Program. Canadian Children, 38, 4-13.

https://doi.org/10.18357/jcs.v38i2.15445

Pak, C. (2007). The Service-Learning Classroom and Motivational Strategies for Learning Spanish: Discoveries from Two Interdisciplinary, Community-Centered Seminars. In A. Wurr, \& J. Hellebrandt (Eds.), Learning the Language of Global Citizenship: Service-Learning in Applied Linguistics. Boston, MA: Anker.

Pellettieri, J. (2011). Measuring Language-Related Outcomes of Community-Based Learning in Intermediate Spanish Courses. Hispania, 94, 285-302.

Pomerantz, E. M., Moorman, E., \& Litwack, S. D. (2007). The How, Whom, and Why of Parents' Involvement in Children's Academic Lives: More Is Not Always Better. Review 
of Educational Research, 77, 379-410. https://doi.org/10.3102/003465430305567

Richards, J. (2012). Teacher Stress and Coping Strategies: A National Snapshot. The Educational Forum (West Lafayette, Ind.), 76, 299-316. https://doi.org/10.1080/00131725.2012.682837

Sheldon, S. B. (2003). Linking School-Family-Community Partnerships in Urban Elementary Schools to Student Achievement on State Tests. The Urban Review, 35, 149165. https://doi.org/10.1023/A:1023713829693

Sheldon, S. B. (2007). Improving Student Attendance with School, Family, and Community Partnerships. The Journal of Educational Research, 100, 267-275. https://doi.org/10.3200/JOER.100.5.267-275

Smith, E. P., Connell, C. M., Wright, G., Sizer, M., Norman, J. M., Hurley, A., \& Walker, S. N. (1997). An Ecological Model of Home, School, and Community Partnerships: Implications for Research and Practice. Journal of Educational and Psychological Consultation, 8, 339-360.

Suarez-Orozco, C., Onaga, M., \& De Lardemelle, C. (2010). Promoting Academic Engagement among Immigrant Adolescents through School-Family-Community Collaboration. Professional School Counseling, 14, 15-26.

Tsung, L., \& Gao, F. (2012). What Accounts for the Underachievement of South Asians in Hong Kong? The Voices of Pakistani and Nepalese Parents. Educational Research, 54, 51-63. https://doi.org/10.1080/00131881.2012.658199

Wehling, S. (2011). Service Learning and Foreign Language Acquisition: Working with the Migrant Community. In C. Wilkerson, \& P. Swanson (Eds.), Got Languages? Powerful Skills for the 21st Century: Dimension 2011-Selected Proceedings of the 2011 Joint Conference of the Southern Conference on Language Teaching, the Louisiana Foreign Language Teachers' Association, and the Southeastern Association of Language Learning Technology. Roswell, GA: Southern Conference on Language Teaching.

Wheeler, L., Guevara, J. R., \& Smith, J. (2018). School-Community Learning Partnerships for Sustainability: Recommended Best Practice and Reality. International Review of Education, 64, 313-337. https://doi.org/10.1007/s11159-018-9717-y 